\title{
Lymphoepithelioma-like gastric carcinoma treated with partial gastrectomy: Two case reports
}

\author{
HUIHUA CAO $^{1}$, JUN XIE $^{2}$, YONGXIANG QIAN ${ }^{3}$, YUGANG WU $^{1}$ and ZHAOQING TANG ${ }^{4}$ \\ Departments of ${ }^{1}$ General Surgery, ${ }^{2}$ Pathology and ${ }^{3}$ Cardiothoracic Surgery, \\ The Third Affiliated Hospital of Soochow University and The First People's Hospital of Changzhou, \\ Changzhou, Jiangsu 213000; ${ }^{4}$ Department of General Surgery, Zhongshan Hospital, \\ Fudan University, Shanghai 200032, P.R. China
}

Received May 6, 2018; Accepted February 21, 2019

DOI: $10.3892 / \mathrm{ol} .2019 .10368$

\begin{abstract}
Lymphoepithelioma-like gastric carcinoma (LELGC) is a rare type of gastric cancer characterized by intense lymphocytic infiltration of the stroma. LELGC is associated with Epstein-Barr virus infection and has a favorable prognosis compared with other types of gastric carcinoma. The clinical symptoms of LELGC are usually similar to those of conventional gastric carcinoma. The diagnosis of LELGC is established based on pathological, histological and immunohistochemical findings. The present report describes the cases of two patients with LELGC who underwent esophagogastroduodenoscopy and computed tomography scans prior to surgery. The two patients accepted surgical treatment, and postoperative pathological and immunohistochemical analyses confirmed LELGC. Neither patient experienced local recurrence or distant metastasis during the postoperative period.
\end{abstract}

\section{Introduction}

Lymphoepithelioma-like gastric carcinoma (LELGC) is a rare type of gastric cancer characterized by lymphocytic infiltration of the tumor stroma (1). Watanabe et al (2) described LELGC for the first time in 1976 (2). LELGC constitutes 1-4\% of all gastric carcinomas $(3,4)$, is considered to predominantly affect males and has a more positive prognosis than other types of gastric carcinoma (5).

Correspondence to: Dr Yugang Wu, Department of General Surgery, The Third Affiliated Hospital of Soochow University and The First People's Hospital of Changzhou, 185 Juqian Street, Changzhou, Jiangsu 213000, P.R. China

E-mail: czyywyg89@163.com

Dr Zhaoqing Tang, Department of General Surgery, Zhongshan Hospital, Fudan University, 180 Fenglin Road, Shanghai 200032, P.R. China

E-mail: tang.zhaoqing@zs-hospital.sh.cn

Key words: Epstein-Barr virus, lymphoepithelioma-like gastric carcinoma, prognosis
LELGC is categorized into 2 subsets: Epstein-Barr virus (EBV)-positive carcinoma and microsatellite instability (MSI)-high carcinoma. EBV-positive cancers commonly comprise increased tumor-infiltrating lymphocytes compared with EBV-negative cancers. MSI-high cancers also present increased tumor-infiltrating lymphocytes compared with non-MSI-high cancers. MSI-high status may result from defective function of DNA mismatch repair enzymes, including MutL homolog 1 or MutS homolog 2, but rarely MutS homolog 6 (6). Overall, $>80 \%$ of LELGC is EBV-positive, whereas the prevalence of MSI-high LELGC is 7-39\%, depending on geographical location (1).

In the present study, 2 cases of LELGC in male patients were examined. The diagnosis and prognosis of LELGC are discussed.

\section{Case report}

Case 1. A 65-year-old man reporting epigastric discomfort for 1 month underwent diagnostic esophagogastroduodenoscopy (EGD) at The Third Affiliated Hospital of Soochow University and The First People's Hospital of Changzhou (Changzhou, China) in August 2016. An ulcerated round lesion of $\sim 4.0 \mathrm{~cm}$ was detected in the lesser curvature of the cardia (Fig. 1A). A pathological examination of a specimen collected with a forceps biopsy indicated that this lesion was a highly atypical hyperplasia and possibly a malignant tumor. A computed tomography (CT) scan revealed that the lesion was a tumor with local ulceration and perigastric lymphadenectasis (Fig. 1B).

Based on the preoperative evaluation, a proximal gastrectomy was performed. The macroscopic lesion was round, with a diameter of $\sim 4.0 \mathrm{~cm}$, and occurred in the cardia and lesser curvature of the stomach. Pathological analysis of the postoperative specimen determined that the lesion was morphologically an ulcerated LELGC invading the subserosal layer, with poor differentiation and lymphovascular invasion (Fig. 1C). In situ hybridization (ISH) performed as previously described (7) revealed that the tumor was negative for Epstein-Barr-encoded RNA (EBER) (Fig. 1D). Immunohistochemical analysis of the postoperative specimen demonstrated that the tumor was positive for receptor tyrosine-protein kinase erbB-2 (c-erbB-2), proliferation marker Ki-67, programmed cell death 1 (PD-1) 
and programmed cell death 1 ligand 1 (PD-L1) (Fig. 2A-D), and the Ki-67 staining index was $\sim 90 \%$. Further immunohistochemical analysis demonstrated that the postoperative specimen was positive for DNA mismatch repair proteins MutS homolog 2, MutS homolog 6 and MutL homolog 1 (Fig. 3A-C), and negative for mismatch repair endonuclease PMS2 (Fig. 3D), which indicated that the tumor was MSI-positive. The resected margins of the specimen were tumor-free and no lymph-node metastasis was detected. The patient was followed up for 18 months and recovered well after surgery, and no sign of local recurrence was detected. This case was one of the only two cases of LELGC treated at The Third Affiliated Hospital of Soochow University and The First People's Hospital of Changzhou during the past several decades, and the MSI characteristics of the tumor were firstly investigated.

Case 2. A 27-year-old man experiencing epigastric pain and a hypodynamic impulse for 1 month, with occasional melena, was admitted to The Third Affiliated Hospital of Soochow University and The First People's Hospital of Changzhou (Changzhou, China) in November 2017. EGD revealed irregular apophyses in the posterior wall of the anterior pyloric region, invading the duodenum, with surface ulceration (Fig. 4A). Pathological examination of a specimen collected with a forceps biopsy confirmed the presence of a gastric antrum cancer, which was considered to be a poorly differentiated adenocarcinoma, but no Helicobacter pylori infection was detected. An enhanced abdominal CT scan identified thickening of the stomach wall in the gastric antrum, with peripheral lymphadenectasis, indicating a malignant tumor (Fig. 4B).

The patient was transferred to Zhongshan Hospital affiliated to Fudan University, (Shanghai, China) where better treatment was available. Following a full preoperative evaluation, a distal gastrectomy was performed. A bulging mass with a central ulcerated lesion $\sim 6.5 \times 5.0 \mathrm{~cm}^{2}$ in size was removed from the lesser curvature of the gastric antrum. Pathological analysis of the postoperative specimen revealed that the lesion had invaded the serous layer, with dense lymphocytic infiltration (Fig. 4C). ISH demonstrated that the tumor cells were positive for EBER (Fig. 4D). Immunohistochemical analysis of the specimen demonstrated that it was positive for pan cytokeratin, Ki-67, PD-1 and PD-L1 (Fig. 5A-D), as well as DNA excision repair protein $\mathrm{ERCC1}$, c-erbB-2, MET proto-oncogene, type II topoisomerase (TOPOII), $\beta$-tubulin, interleukin- 9 , vimentin, keratin 20 and keratin 19, but that it was negative for FYN proto-oncogene, neural adhesion molecule 1, glycoprotein hormone $\alpha$, tumor protein p63 and keratin 7 . The ERCC1, Ki-67, MET and TOPOII staining indices were $\sim 90,60,90$ and 3\%, respectively. Morphologically and immunohistochemically, the lesion was characterized as LELGC. The resected margins were tumor-free and no lymph-node metastasis was detected. Following surgery, the patient was treated with tegafur, gimeracil and oteracil potassium. This medicine was taken at the dose of $40 \mathrm{mg}$, twice daily for three weeks and stopped for one week, with a cycle of four weeks and for a total of one year. The patient recovered well during the 4-month follow-up after surgery, with no sign of local recurrence. In addition, during the follow-up, serum analysis revealed that the patient was positive for EBV antigen.

Histology and immunohistochemistry examinations. Specimens from case 1 and case 2, including gastroscopic biopsy specimens and postoperative specimens, were sent for analysis. The postoperative specimen from case 2 was analyzed in the Zhongshan Hospital affiliated to Fudan University (Shanghai, China), whereas the other specimens from cases 1 and 2 were all analyzed at The Third Affiliated Hospital of Soochow University and The First People's Hospital of Changzhou (Changzhou, China). All specimens were analyzed following the same protocol in the two hospitals. Specimens were fixed in $10 \%$ formalin for $24 \mathrm{~h}$ at room temperature and embedded in paraffin. Sections were cut into $4-\mathrm{mm}$ thick slices. For histological examination, sections were subjected to routine deparaffinization using xylene (twice for $10 \mathrm{~min}$ ) and rehydration with ethanol (twice for $5 \mathrm{~min}$ each, including 95\% ethanol for $5 \mathrm{~min}$ and $70 \%$ ethanol for $5 \mathrm{~min}$ (Shanghai Sangong Pharmaceutical Co., Ltd., Shanghai, China). Sections were washed in distilled water for $1 \mathrm{~min}$ and stained with $99 \%$ hematoxylin and eosin for $10 \mathrm{~min}$ at room temperature (Shanghai Sangong Pharmaceutical Co.), and mounted with xylene-based mounting medium (Shanghai Sangong Pharmaceutical Co.). For immunohistochemical examination, sections were microwaved for $10 \mathrm{~min}$ in $0.01 \mathrm{~mol} / 1$ citrate buffer (Shanghai Yu Bo Biological Technology Co. Ltd., Shanghai, China) for antigen retrieval, and allowed to cool for $30 \mathrm{~min}$. Endogenous peroxidase activity was quenched by incubating sections with 3\% hydrogen peroxide (Shanghai Yu Bo Biological Technology Co. Ltd.) in methanol for $10 \mathrm{~min}$. Non-specific binding was blocked by incubation with $5 \%$ bovine serum albumin (Shanghai Acme Biochemical Co. Ltd., Shanghai, China) diluted in PBS (Beijing TransGen Biotech Co. Ltd.) for $10 \mathrm{~min}$ at room temperature. Sections were washed three times with PBS, and incubated at $4^{\circ} \mathrm{C}$ overnight with murine antihuman monoclonal antibodies. Antibodies used for case 1 were as follows: Anti-erbB-2 (1:200; Clone EP1045Y; Abcam, Cambridge, UK), anti-ki-67 (1:200; Clone SP6; Abcam), anti-PD-1 (1:200; Clone SP269; Abcam), anti-PD-L1 (1:200; Clone 73-10; Abcam), anti-MSH2 (1:200; Clone 3A2B8C; Abcam), anti-MSH6 (1:200; Clone EPR20316; Abcam), anti-MLH1 (1:200; Clone EPR3894; Abcam) and anti-PMS2 (1:200; Clone EPR3947; Abcam). Antibodies used for case 2 were as follows: Anti-pan cytokeratin (1:200; Clone C-11; Abcam), anti-Ki-67 (1:200; Clone EPR3610, Abcam), anti-PD-1 (1:200; Clone NAT105, Abcam) and anti-PD-L1 (1:200; Clone 28-8, Abcam). Sections were then incubated with an anti-mouse immunoglobulin G2b-PE peroxidase for $30 \mathrm{~min}$ at room temperature $(1: 1,000$; F1032; SouthernBiotech, Birmingham, AL, USA). Signal was established following incubation with 3,3-diaminobenzidine tetrahydrochloride diluted in Tris-HCl buffer ( $\mathrm{pH}$ 7.6) containing $0.02 \%$ hydrogen peroxide for $10 \mathrm{~min}$ at room temperature (Shanghai XY Biotechnology Co. Ltd.). Sections were finally counterstained with hematoxylin and mounted as aforementioned. Sections that were not stained with primary antibodies represented the negative controls. 
A

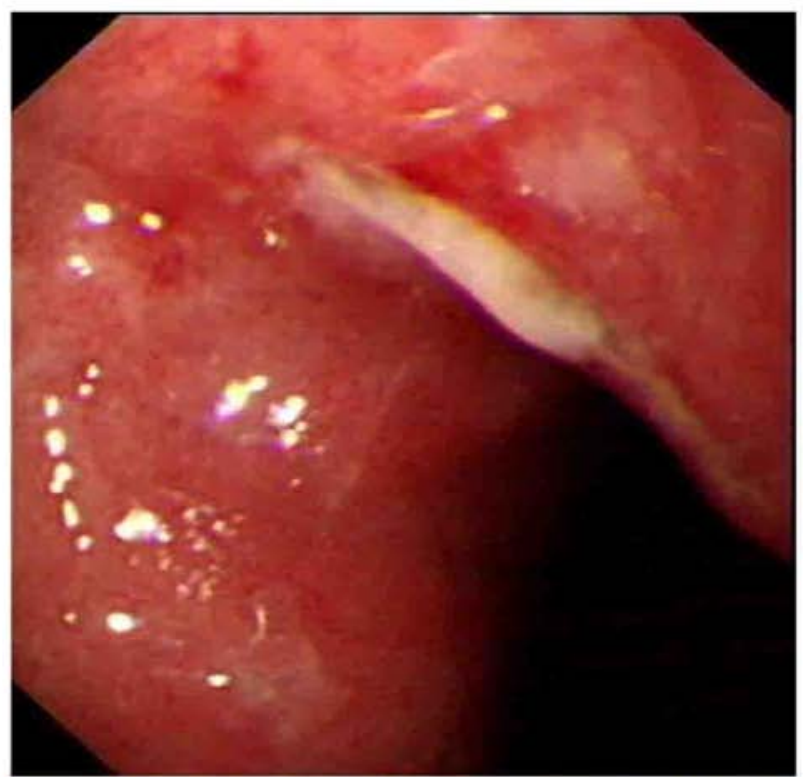

C

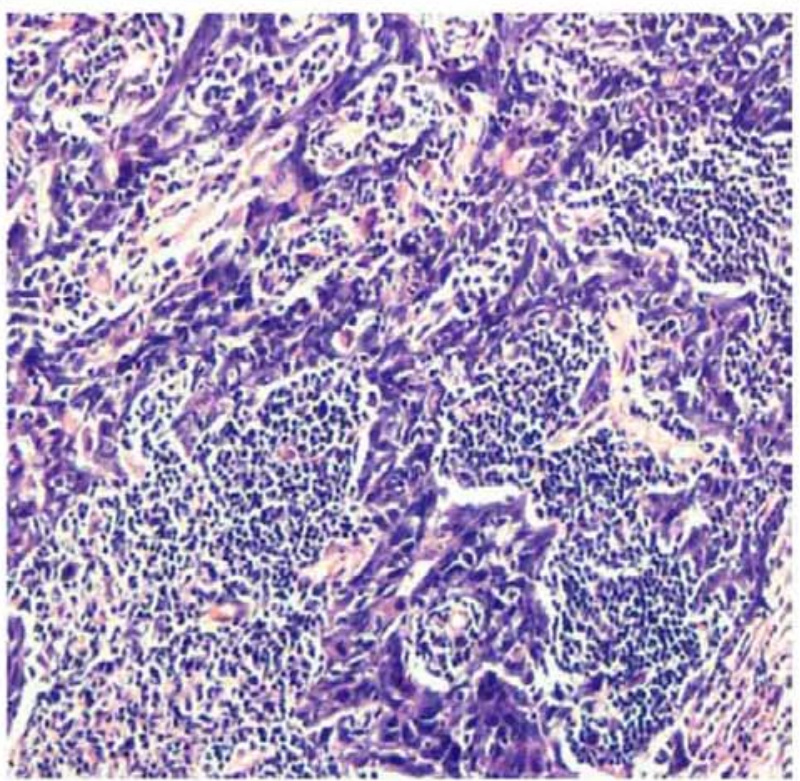

$\mathrm{B}$

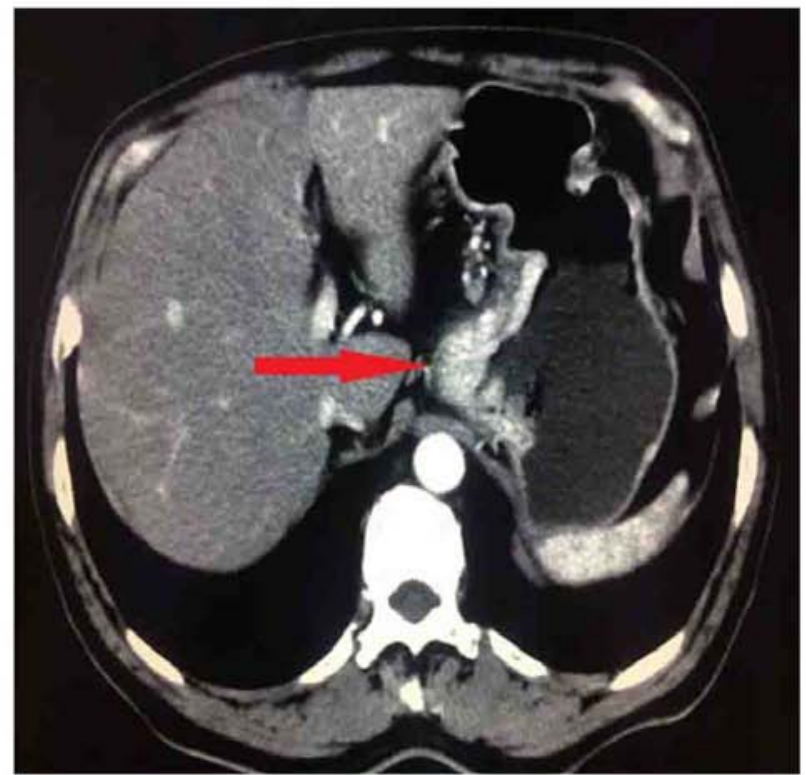

D

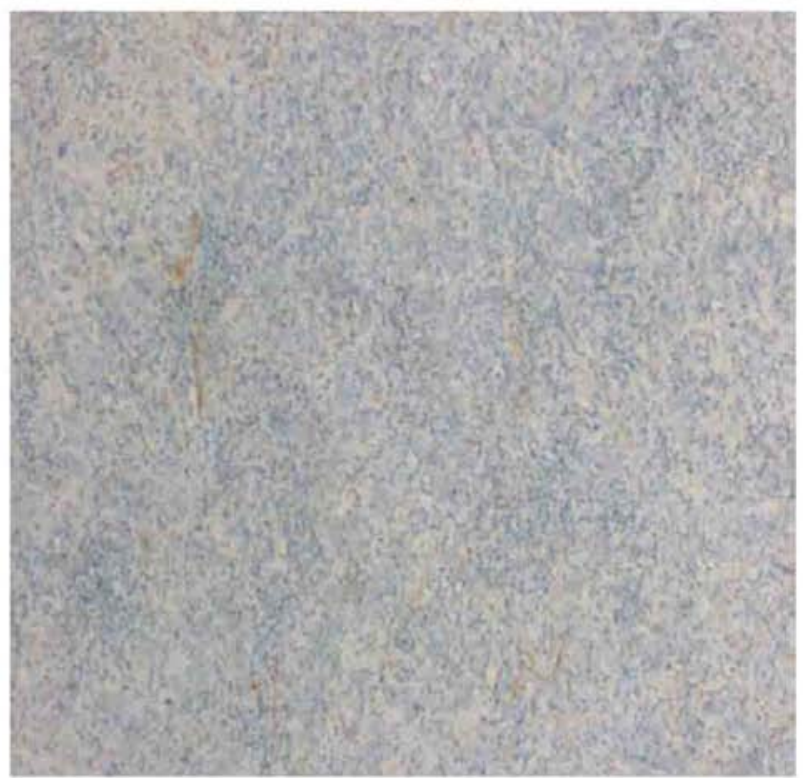

Figure 1. Characteristics of the tumor from patient 1. (A) A large, round, ulcerated lesion in the lesser curvature of the cardia measuring $\sim 4.0 \mathrm{~cm}$, as revealed by esophagogastroduodenoscopy. (B) Tumor in the cardia (highlighted by the red arrowhead) with local ulceration and perigastric lymphadenectasis observed by abdominal computed tomography scan. (C) Dense lymphocytic infiltration of the tumor stroma, revealed by hematoxylin and eosin staining of the postoperative specimen (x200 magnification). (D) In situ hybridization for Epstein-Barr-virus-encoded RNA (x100 magnification).

All sections were observed using an Olympus BX43 microscope (magnification, x100 or x200; Olympus Corporation, Tokyo, Japan).

\section{Discussion}

LELGC is a type of gastric carcinoma with typical clinicopathological characteristics $(8,9)$, including a chiseled tumor margin, dense lymphocytic infiltration with the number of infiltrating lymphocytes exceeding the number of tumor cells, obscure cytoplasmic borders, a syncytial growth pattern with a poorly formed glandular structure and no desmoplasia (10). LELGC is also known as 'gastric carcinoma with lymphoid stroma (2), and has been reported to occur in various organs, including the stomach, salivary gland, thymus, larynx, lung, esophagus, cervix and skin (1).
At present, there are several similar studies concerning LELGC; however, the current study presents novel information and therefore remains of significant value. During the past several decades, these were the only 2 cases of LELGC on record in The Third Affiliated Hospital of Soochow University and The First People's Hospital of Changzhou, indicating the rarity of this type of gastric cancer. For the EBV-negative patient 1, further immunohistochemical analysis was performed to investigate the MSI characteristics of the tumor. Additionally, the EBV antigen was tested in serum from EBV-positive patient 2. The therapeutic strategies for EBV-associated LELGC are also discussed in the present study.

EBV has an etiological association with LELGC and the prevalence of EBV-positive gastric cancer is reportedly $8.29 \%(11,12)$. EBV infection is also associated with 

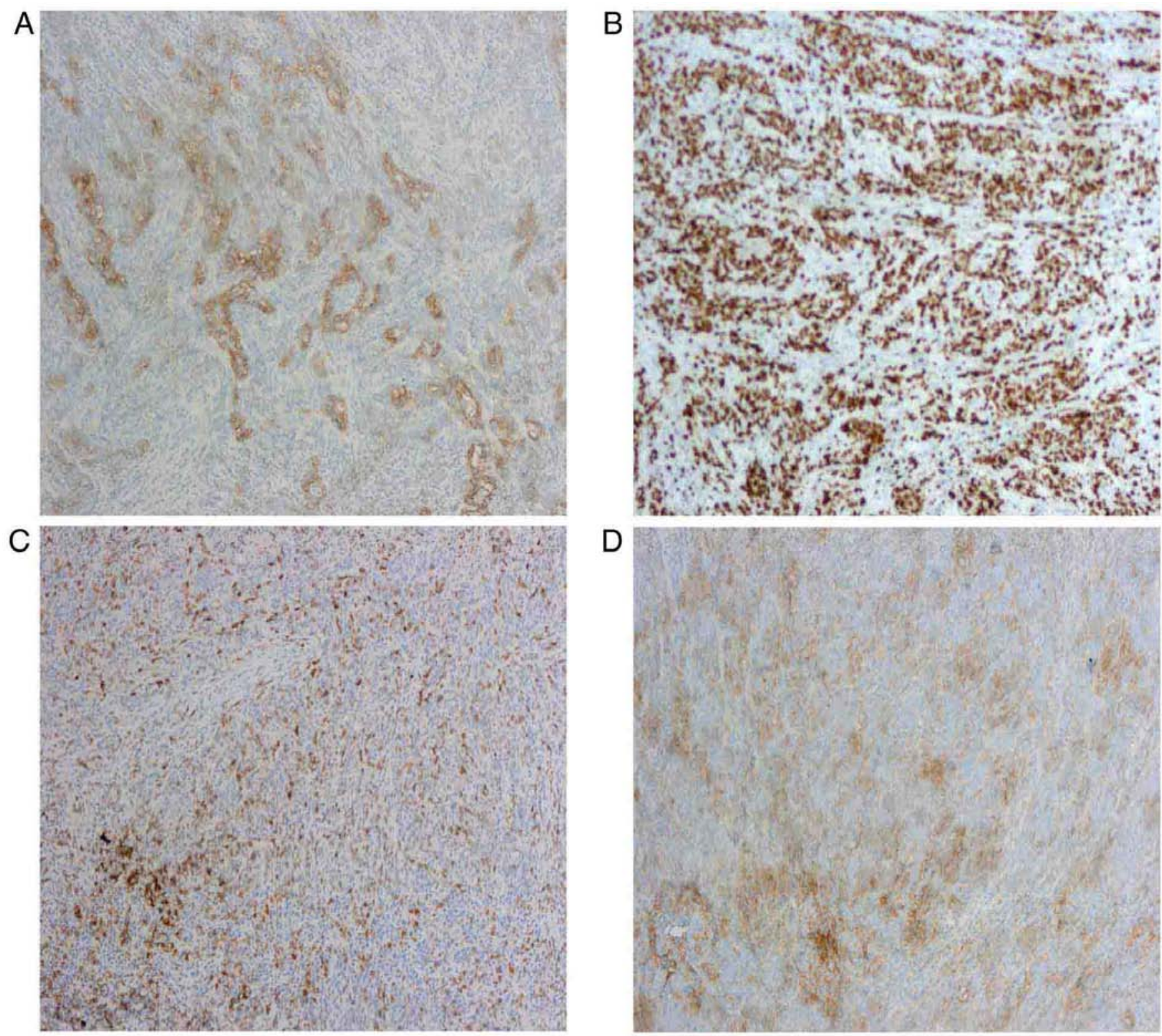

Figure 2. Immunohistochemical staining of the tumor from patient 1. (A) Receptor tyrosine-protein kinase erbB-2, (B) proliferation marker Ki-67, (C) programmed cell death 1 and (D) programmed cell death ligand 1 (x100 magnification). The Ki-67 staining index was $\sim 90 \%$.

nasopharyngeal carcinomas. However, the mechanism by which EBV contributes to the carcinogenesis of the gastric mucosa remains unknown (13-15). The presence of EBV in tumor cells was confirmed with ISH targeting EBER. In the present study, the tumor cells in case 1 were EBV-negative and MSI-positive, whereas those in case 2 were EBV-positive. In the postoperative follow-up review, patient 2 was also found to be positive for serum EBV antigen.

The clinical symptoms of LELGC are usually similar to those of conventional gastric carcinoma, and include abdominal pain, loss of appetite and weight loss $(10,13)$. However, LELGC, unlike conventional gastric carcinoma, is usually located in the proximal stomach and occurs predominantly in males (5). LELGC is also easily confused macroscopically with submucosal tumors, as it often presents as an ulcerated tumor with a thickened gastric wall $(5,8)$. Therefore, a definitive diagnosis may be difficult based on an endoscopic biopsy prior to surgery. The presentation of LELGC on CT can also vary, appearing as a thickened focal mucosa, apparent thickening of the gastric wall with contrast enhancement or a bulky mass (16). Therefore, it is difficult to distinguish LELGC from lymphoma, gastrointestinal stromal tumors, glomus tumors and neurogenic tumors with CT alone $(4,5)$. However, an accurate diagnosis can be achieved based on the histological characteristics of the dissected specimen following curative surgery or endoscopic submucosal dissection (ESD). Fukayama and Ushiku (17) concluded that the diagnosis of EBV-positive LELGC should be based on dysplastic epithelial cells and the detection of EBER with ISH. Shinozaki-Ushiku et al (18) reported that immunohistochemistry with an antibody directed against cytokeratin and EBER-targeting ISH detected EBV-associated LELGC. The 2 patients in the current study presented with histologically dense lymphoid cell infiltration of the stroma, which is consistent with the pathological features of LELGC. Patient 2 was also positive for EBER and met the diagnostic criteria for EBV-positive LELGC. 

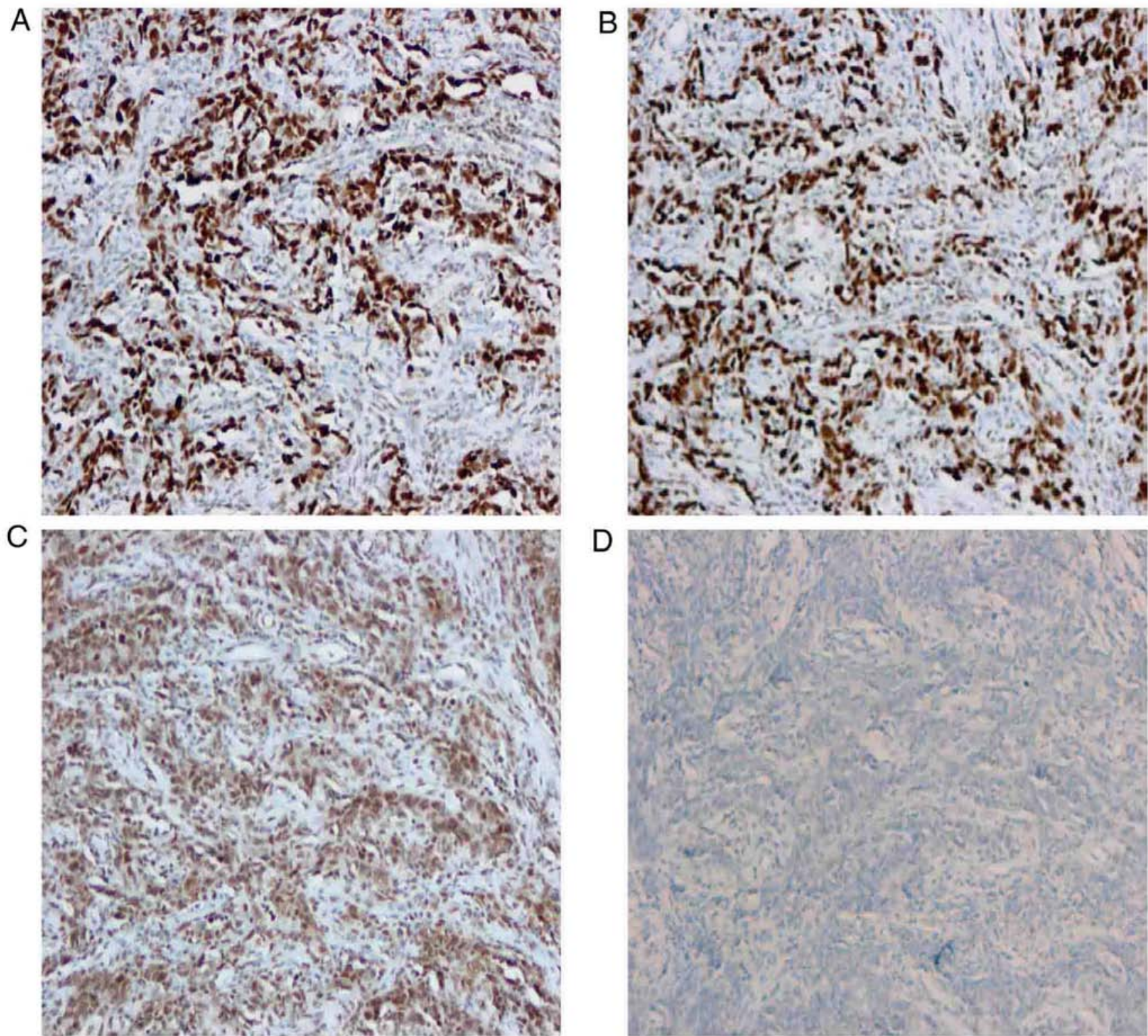

Figure 3. Immunohistochemical staining for mismatch repair proteins in the tumor from patient 1. (A) MutS homolog 2, (B) MutS homolog 6, (C) MutL homolog 1 and (D) mismatch repair endonuclease PMS2 (x200 magnification).

In the present study, the 2 patients chose laparotomy for the treatment of their advanced-stage tumors. A proximal gastrectomy was performed in patient 1 as the lesion was large and had invaded the subserosa. It was necessary to dissect at least $3 \mathrm{~cm}$ into the transhiatal esophagus and to further confirm the negative margin by frozen section. In patient 2 , the lesion was in the gastric antrum, so a distal gastrectomy was performed. However, other surgical methods have been reported in previous studies. According to Lee et al (8), EBV-associated early LELGC was diagnosed by ESD, with a favorable long-term outcome. Chen et al (1) also reported that laparoscopic surgery can be used for the diagnosis and treatment of LELGC, and that ESD can be considered for early EBV-positive LELGC, particularly in patients with a serious comorbidity or a high surgical risk. In the present study, in addition to surgical resection, postoperative chemotherapy was administered to patient 2 , who is currently on tegafur, gimeracil and oteracil potassium treatment. Patient 1 rejected our advice to receive chemotherapy. This chemotherapy strategy was in accordance with the therapeutic strategies for EBV-associated lymphoepithelioma-like carcinoma reported in the study by Tse and Kwong (19), which concluded that EBV-targeted therapy is important and that the prophylactic use of antiviral drugs is effective in reducing the occurrence of EBV-positive lymphoproliferative diseases. Geng and Wang (20) reported several similar therapeutic strategies for lymphoproliferative diseases, including novel antivirals, immunotherapy and geneor pathway-targeted therapies.

As aforementioned, patients with LELGC have a relatively more positive prognosis than those with conventional gastric carcinoma. Tak et al (21) reported that postoperative recurrence or metastasis tended to occur less in patients with LELGC than in patients with poorly differentiated gastric carcinomas. Park et al (22) demonstrated that the 5-year survival rate of patients with LELGC was higher than that of patients with non-LELGC (97.7 vs. $89.4 \%$ ). A similar 

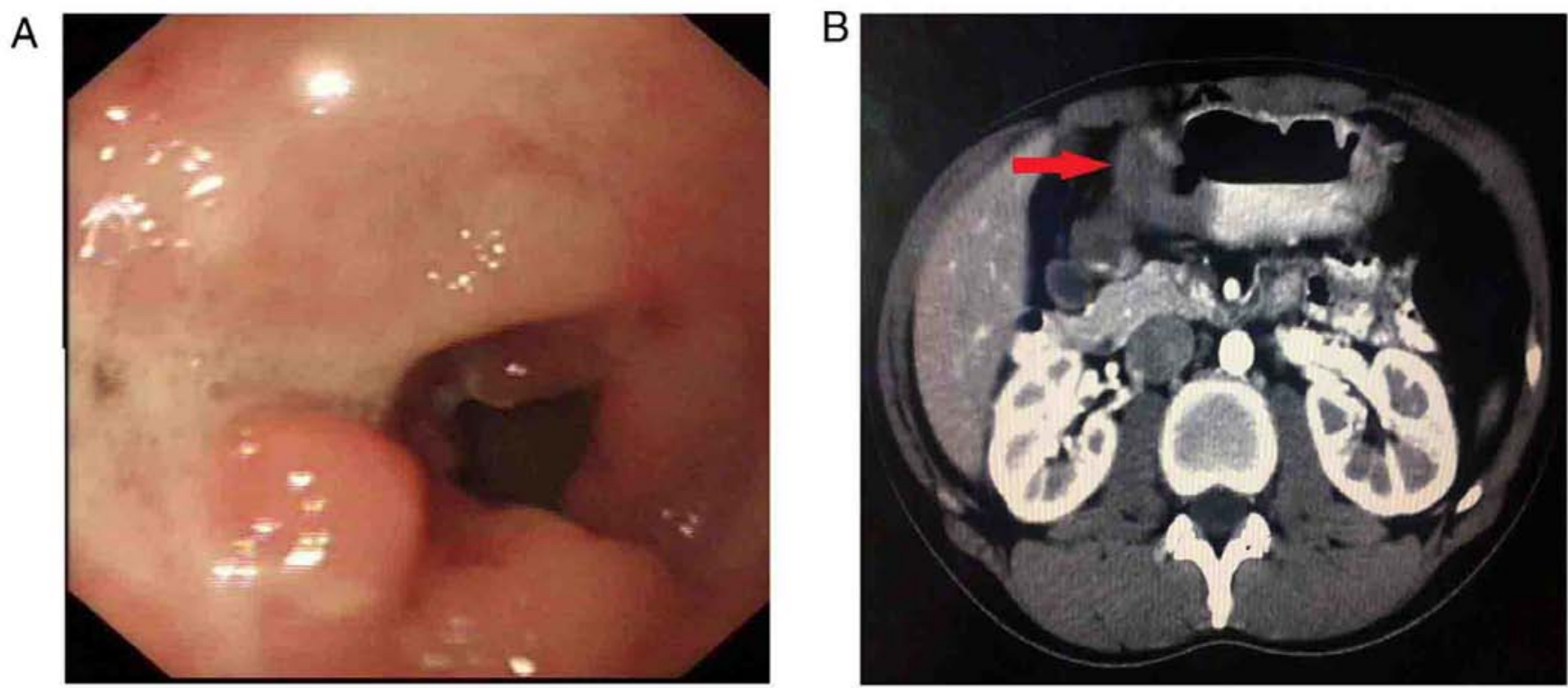

C

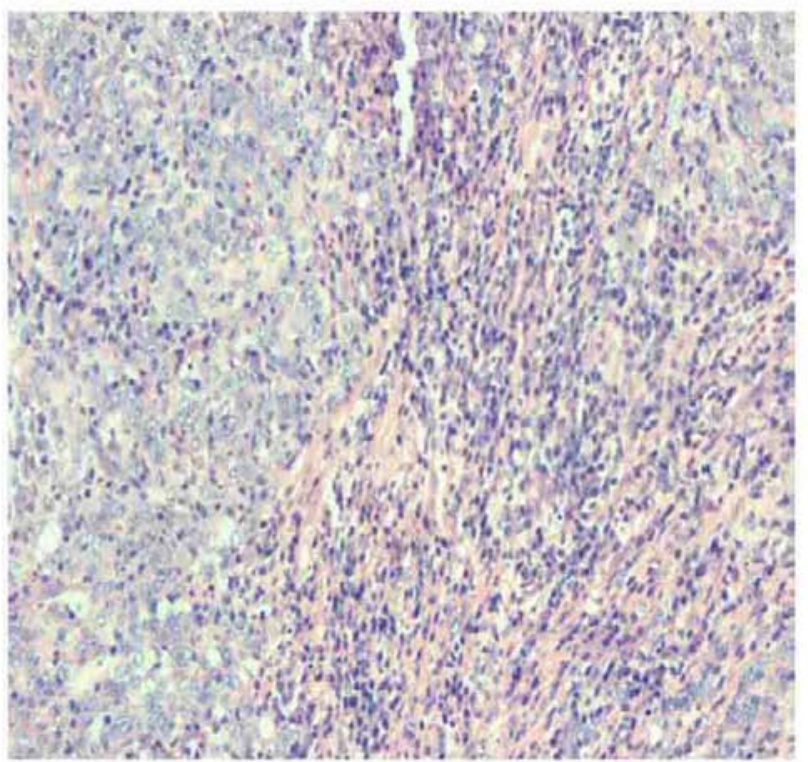

D

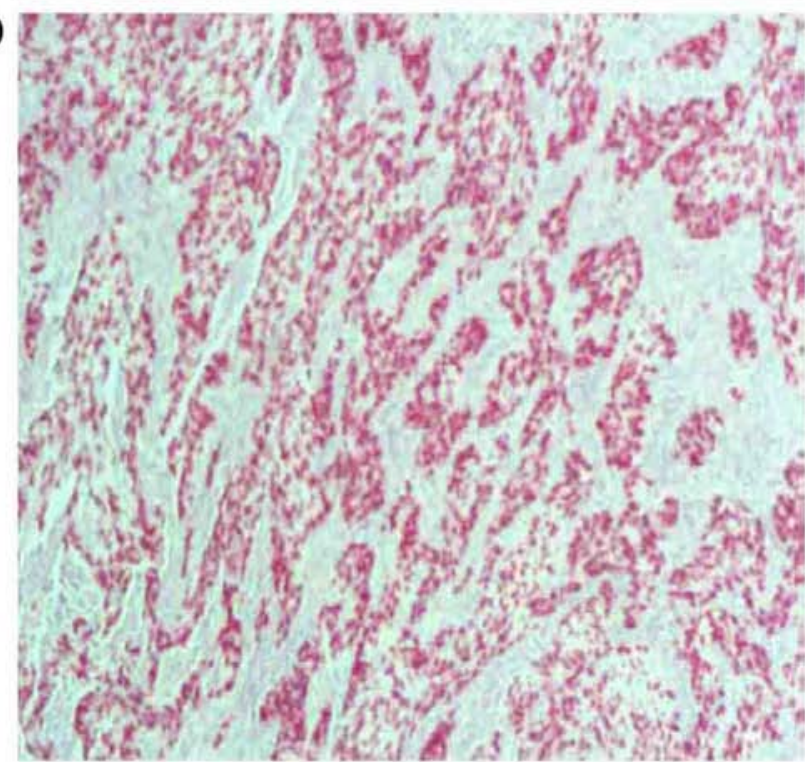

Figure 4. Characteristics of the tumor from patient 2. (A) Irregular apophyses in the posterior wall of the anterior pyloric region invading the duodenum with surface ulceration, as revealed by esophagogastroduodenoscopy. (B) Thickening of the stomach wall in the gastric antrum (highlighted by the red arrowhead), with peripheral lymphadenectasis, as observed by abdominal computed tomography scan. (C) Dense lymphocytic infiltration of the tumor stroma, revealed by hematoxylin and eosin staining of the postoperative specimen (x200 magnification). (D) In situ hybridization for Epstein-Barr-virus-encoded RNA (x100 magnification)

conclusion was drawn in the study by Nakamura et al (23), which revealed that the 5-year survival rate of patients with LELGC following surgical treatment was higher than that of patients with conventional adenocarcinoma (84 vs. 58\%). In the present study, patient 1 appeared to exhibit symptoms of reflux owing to the proximal gastrectomy. Fortunately, the reflux could be well controlled by medication. Overall, the 2 patients underwent laparotomy and exhibited good outcomes during follow-up, despite the deep invasion of the tumor cells. However, the follow-up period was short, and the long-term effects of this treatment require further examination. A number of studies have investigated why patients with LELGC exhibit a higher survival rate than patients with other forms of gastric cancer. Lee et al (8) reported that LELGC is characterized by dense lymphocytic infiltration of the tumor stroma, which could be associated with the positive prognosis.
A massive lymphocytic reaction could prevent the spread of the tumor through the gastric wall $(24,25)$. Song et al (10) previously reported that the 12-year disease-free survival rate of LELGC patients was $\sim 95 \%$, and suggested that the extensive infiltration of lymphocytes contributed to low tumor metastasis and an improved prognosis.

In conclusion, 2 cases of LELGC, a rare stomach neoplasm that is associated with EBV infection, are described in the present study. It is difficult to distinguish LELGC from ordinary gastric carcinoma with an endoscopic biopsy, as the stromal lymphocytic infiltration is dense. A diagnosis of LELGC should be established based on the pathological, histological and immunohistochemical analyses of the postoperative specimen. LELGC generally has a more positive prognosis than other types of EBV-positive gastric carcinomas or conventional gastric carcinomas. 

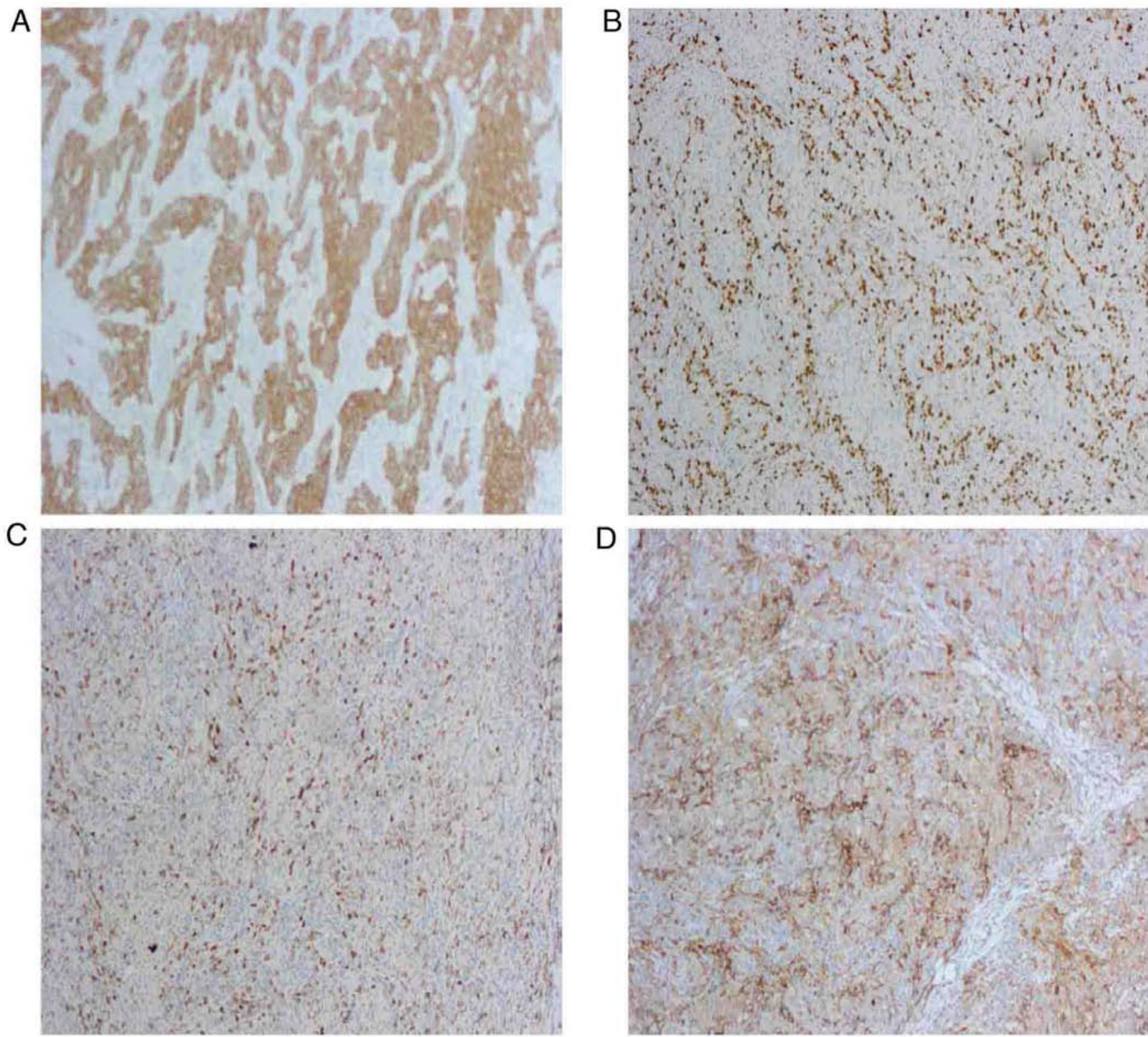

Figure 5. Immunohistochemical staining of the tumor from patient 2. (A) Pan cytokeratin, (B) proliferation marker Ki-67, (C) programmed cell death 1 and (D) programmed cell death ligand 1 (x100 magnification). The Ki-67 staining index was $~ 60 \%$.

\section{Acknowledgements}

The authors would like to thank Dr Zhaoli Li (Department of Pathology, The Third Affiliated Hospital of Soochow University and The First People's Hospital of Changzhou, Changzhou, China) for her assistance in immunohistochemical staining analyses.

\section{Funding}

The present study was supported by a Changzhou Municipal Scientific Research grant (no. CE20125020).

\section{Availability of data and materials}

The datasets used and/or analyzed during the current study are available from the corresponding author on reasonable request.

\section{Authors' contributions}

HC, YW and ZT wrote the manuscript and analyzed the clinicopathological data. JX and YQ performed the follow-up and collected the clinicopathological data of the patients. YW and $\mathrm{ZT}$ assisted $\mathrm{HC}$ to revise the manuscript, and $\mathrm{YW}$ assisted to fund the study.

\section{Ethics approval and consent to participate}

This study was approved by the Ethics Committee of The Third Affiliated Hospital of Soochow University.

\section{Patient consent for publication}

Written consent for publication was provided by the patients included in this study. 


\section{Competing interests}

The authors declare that they have no competing interests.

\section{References}

1. Chen M, Yin L, Yao Y, Wang L, Xu G, Zhang X, Lv Y, Sun QI, Fan $\mathrm{X}$ and Zou X: Lymphoepithelioma-like gastric carcinoma in a patient with rectal laterally spreading tumor: A case report. Oncol Lett 11: 2491-2496, 2016.

2. Watanabe H, Enjoji M and Imai T: Gastric carcinoma with lymphoid stroma. Its morphologic characteristics and prognostic correlations. Cancer 38: 232-243, 1976.

3. Corvalan A, Ding S, Koriyama C, Carrascal E, Carrasquilla G, Backhouse C, Urzua L, Argandona J, Palma M, Eizuru Y and Akiba S: Association of a distinctive strain of Epstein-Barr virus with gastric cancer. Int J Cancer 118: 1736-1742, 2006.

4. Kim SW, Shin HC, Kim IY, Kim CJ, Lee JH, Lee CK and Jeong DJ: Epstein-Barr virus-associated lymphoepithelioma-like gastric carcinoma presenting as a submucosal mass: CT findings with pathologic correlation. Korean J Radiol 11: 697-700, 2010.

5. Xu Q, Du J and Liu BL: Lymphoepithelioma-like gastric carcinoma located in the lesser curvature of the gastric body: A case report and review of the literature. Mol Clin Oncol 4: 405-408, 2016.

6. Grogg KL, Lohse CM, Pankratz VS, Halling KC and Smyrk TC: Lymphocyte-rich gastric cancer: Associations with Epstein-Barr virus, microsatellite instability, histology, and survival. Mod Pathol 16: 641-651, 2003.

7. Weiss LM and Movahed LA: In situ demonstration of Epstein-Barr viral genomes in viral-associated B cell lymphoproliferations. Am J Pathol 134: 651-659, 1989.

8. Lee JY, Kim KM, Min BH, Lee JH, Rhee PL and Kim JJ: Epstein-Barr virus-associated lymphoepithelioma-like early gastric carcinomas and endoscopic submucosal dissection: Case series. World J Gastroenterol 20: 1365-1370, 2014.

9. Cheng N, Hui DY, Liu Y, Zhang NN, Jiang Y, Han J, Li HG, Ding YG, Du H, Chen JN and Shao CK: Is gastric lymphoepithelioma-like carcinoma a special subtype of EBV-associated gastric carcinoma? New insight based on clinicopathological features and EBV genome polymorphisms. Gastric Cancer 18: 246-255, 2015

10. Song HJ, Srivastava A, Lee J, Kim YS, Kim KM, Ki Kang W, Kim M, Kim S, Park CK and Kim S: Host inflammatory response predicts survival of patients with Epstein-Barr virus-associated gastric carcinoma. Gastroenterology 139: 84-92.e82, 2010.

11. Shibata D, Tokunaga M, Uemura Y, Sato E, Tanaka S and Weiss LM: Association of Epstein-Barr virus with undifferentiated gastric carcinomas with intense lymphoid infiltration. Lymphoepithelioma-like carcinoma. Am J Pathol 139: 469-474, 1991.

12. Sousa H, Pinto-Correia AL, Medeiros R and Dinis-Ribeiro M: Epstein-Barr virus is associated with gastric carcinoma: The question is what is the significance? World J Gastroenterol 14: 4347-4351, 2008.
13. Wang ZH, Zhao JJ and Yuan Z: Lymphoepithelioma-like gastric carcinoma: A case report and review of the literature. World J Gastroenterol 22: 3056-3061, 2016.

14. Chang MS, Kim WH, Kim CW and Kim YI: Epstein-Barr virus in gastric carcinomas with lymphoid stroma. Histopathology 37: 309-315, 2000.

15. Kang GH, Lee S, Kim WH, Lee HW, Kim JC, Rhyu MG and Ro JY: Epstein-Barr virus-positive gastric carcinoma demonstrates frequent aberrant methylation of multiple genes and constitutes $\mathrm{CpG}$ island methylator phenotype-positive gastric carcinoma. Am J Pathol 160: 787-794, 2002.

16. MaedaE,Akahane M,Uozaki H,Kato N,Hayashi N,Fukayama M and Ohtomo K: CT appearance of Epstein-Barr virus-associated gastric carcinoma. Abdom Imaging 34: 618-625, 2009.

17. Fukayama $M$ and Ushiku T: Epstein-Barr virus-associated gastric carcinoma. Pathology, Res Pract 207: 529-537, 2011.

18. Shinozaki-Ushiku A, Kunita A and Fukayama M: Update on Epstein-Barr virus and gastric cancer (review). Int J Oncol 46: 1421-1434, 2015.

19. Tse E and Kwong YL: Epstein Barr virus-associated lymphoproliferative diseases: The virus as a therapeutic target. Exp Mol Med 47: e136, 2015.

20. Geng L and Wang X: Epstein-Barr virus-associated lymphoproliferative disorders: Experimental and clinical developments. Int J Clin Exp Med 8: 14656-14671, 2015.

21. Tak DH, Jeong HY, Seong JK, Moon HS and Kang SH: Comparison of clinical characteristics and prognostic factors between gastric lymphoepithelioma-like carcinoma and gastric adenocarcinoma. Korean J Gastroenterol 62: 272-277, 2013.

22. Park S, Choi MG, Kim KM, Kim HS, Jung SH, Lee JH, Noh JH, Sohn TS, Bae JM and Kim S: Lymphoepithelioma-like carcinoma: A distinct type of gastric cancer. J Surg Res 194: 458-463, 2015.

23. Nakamura S, Ueki T, Yao T, Ueyama $\mathrm{T}$ and Tsuneyoshi $\mathrm{M}$ : Epstein-Barr virus in gastric carcinoma with lymphoid stroma. Special reference to its detection by the polymerase chain reaction and in situ hybridization in 99 tumors, including a morphologic analysis. Cancer 73: 2239-2249, 1994.

24. Liu S, Jin L, Xu X, Lin N, Lei B and Shen H: Pathological and computed tomography findings of lymphoepithelioma-like gastric carcinoma with epithelioid granulomas: A case report. Oncol Lett 5: 549-551, 2013.

25. Tamura T, Hamada T, Sako T, Makihara K, Yamada K, Kashima K, Yokoyama S, Hirata K, Hachiya Y, Fukuyama T and Hirano Y: Lymphoepithelioma-like carcinoma of the stomach with epithelioid granulomas. Case Rep Gastroenterol 4: 361-368, 2010.

This work is licensed under a Creative Commons Attribution-NonCommercial-NoDerivatives 4.0 International (CC BY-NC-ND 4.0) License. 